\title{
Simulation of Power System Substation Communications Architecture Based on IEC 61850 Standard
}

\author{
Mohammad Golshani \\ mohammad.golshani@brunel.ac.uk
}

\author{
Gareth A. Taylor \\ gareth.taylor@brunel.ac.uk
}

\author{
Ioana Pisica \\ ioana.pisica@brunel.ac.uk
}

Brunel Institute of Power Systems, Brunel University, London, UK

\begin{abstract}
Changes in the wholesale electricity market alongside the difficulties in upgrading the transmission system have increased the complexity of power networks operations. This fact has placed heavier demands on developing new technologies to manage power systems reliably. Driven by the growing smart grid applications, ICT infrastructures are becoming more important for the communication of monitoring, control and protection information at both the local and wide area levels. IEC 61850 is a standard released by the International Electrotechnical Commission (IEC) for power utilities that can provide integrated and interoperable data communications. It defines communication between Intelligent Electronic Devices (IEDs) in substations and related system requirements. Although it was originally introduced for the automation of substations, the application of IEC61850 is expanding rapidly. In this paper, we present simulation and modelling of a typical power system substation communication infrastructure. Furthermore, the deployment of IEC 61850 for Wide Area Network (WAN) applications, such as Phasor Measurement Units (PMUs), will be investigated.
\end{abstract}

Index Terms--Data communications, IEC 61850, IED, OMNeT++, PMU, Smart Grid, Substation Automation.

\section{INTRODUCTION}

Substations are key nodes in the power system network, where information from the system is retrieved and used for the reliable operation and management. Substation Automation Systems (SAS) are now being implemented using IEDs interconnected through communication network technologies to facilitate the substation monitoring, control and protection. The interest on SAS has been increasing rapidly due to its numerous benefits to utilities. Digital data acquisition affords a level of visibility never considered possible in the electromechanical era of substations. Also SAS provides additional capabilities and information that can be used to further improve operations, maintenance, and efficiencies in substations [1], [2].

As a consequence of employing advanced and fast devices, the efficient and high-speed communication infrastructure has become an important issue in the design of substations. In this regard, the state-of-the-art IEC 61850 standard has enabled IEDs and devices in a substation to be integrated on a high speed communication network. On the other hand, IEC 61850 has the objective of enabling interoperability between IEDs within a substation. Interoperability is defined as the ability of two or more IEDs from the same vendor, or different vendors, to exchange information and use that information for correct execution of specified functions [3]. By applying object oriented (OO) data and service models, IEC 61850 support all substation functions and provides more flexibility to the developer and users [4].

Due to the criticality of some of the smart grid's applications, the communication infrastructures performance in such networks need a thorough analysis to ascertain that the required specifications will be met. In this research, for the performance analysis of substation communications network typical substation communication architecture has been simulated using $\mathrm{OMNeT}++$, an open source Discrete Event Simulation (DES) tool [5]. DES can provide a flexible, scalable and highly repeatable way to investigate and study the behaviour of the communication networks under different conditions. The purpose of this research is to create IEC 61850-based IED models and setup a simulation framework for substation communication network. One of the important aspects of communication networks performance analysis is the delay characteristics. Especially for the smart grid's control and protection functions that have a fast response time requirement. By simulating the substation communications network using DES tools the End-to-End (EtE) delay of the specific transmitted information can be investigated in detail.

Furthermore, in this research we performed a preliminary analysis on introducing IEC 61850 protocol for PMU communications over substation level. Basically, PMUs are deployed for Wide Area Monitoring Systems (WAMS). A PMU-based WAMS is a system in which PMUs measure and time-stamp power system parameters. Then measured data from geographically distributed PMUs are transmitted to central location called Phasor Data Concentrator (PDC). The PDC collects data and produce a real-time and time-aligned output data stream that can be exploited by smart grid applications. Although PMUs transmit data from substations to PDC through WAN, investigating their behaviour inside the substation can be an effective step in introducing new protocol for their communications [6]. 
The rest of the paper is structured as follows: Section II describes the Substation Automation System. Section III provides the relevant background on IEC 61850. The simulation of the substation communications architecture using OMNeT++ is presented in Section IV. Finally, the paper is concluded in Section V.

\section{SUbSTATION AUTOMATION SYSTEM}

It is not sufficient that substations operate properly; they must also operate reliably under credible contingency situations [7]. Fast and reliable information about the current state of the power system leads to better operation and management. In this regard, SAS can provide powerful, fast, and viable way to design, implement, and automate substation monitoring, protection, and control functions in modern transmission and distribution grids [8]. The deployment of SAS has also increased to fulfil a market requirement to decrease the total cost. For example, optimization of the maintenance cost and, in turn, reducing the life cycle costs of substation, provide highly efficient operation or near-limit operation of substation equipment, etc. [9]. Automated substations consist of smart and advanced equipment, such as relays, circuit breakers, transformers, switches, etc. that are integrated and monitored by a graphical interface unit that can be remotely accessed [10]. During the last decades, electromechanical devices in SAS have been replaced by IEDs and most functions including protection and control are now performed by them. The basic functions of a SAS can be categorized as described in Table I. Most SAS have these functions even though these may vary in different projects [9], [11].

The architecture of the SAS can be mapped into three levels hierarchically as shown in Fig. 1 [12]. Station level,

TABLE I

OVERVIEW OF SAS FUNCTIONS

\begin{tabular}{|c|c|}
\hline $\begin{array}{c}\text { Basic } \\
\text { Functions }\end{array}$ & Examples \\
\hline Monitoring & $\begin{array}{l}\text { - Monitoring of Switchgear status, status of } \\
\text { transformer and tap changer, status of protection and } \\
\text { control equipment, etc. } \\
\text { - Monitoring of electrical parameters; e.g. frequency, } \\
\text { voltage, current, real and reactive power, etc. } \\
\text { - Facility fault and device disturbance record }\end{array}$ \\
\hline Control & $\begin{array}{l}\text { - Control of switchgear and transformer tap } \\
\text { - Synchronism check and interlocking } \\
\text { - Load shedding, voltage regulation, reactive power } \\
\text { control, etc }\end{array}$ \\
\hline Protection & $\begin{array}{l}\text { - Protection for transmission line, transformer, busbar, } \\
\text { Feeder, etc. } \\
\text { - Overcurrent, distance, differential protections, etc. }\end{array}$ \\
\hline
\end{tabular}

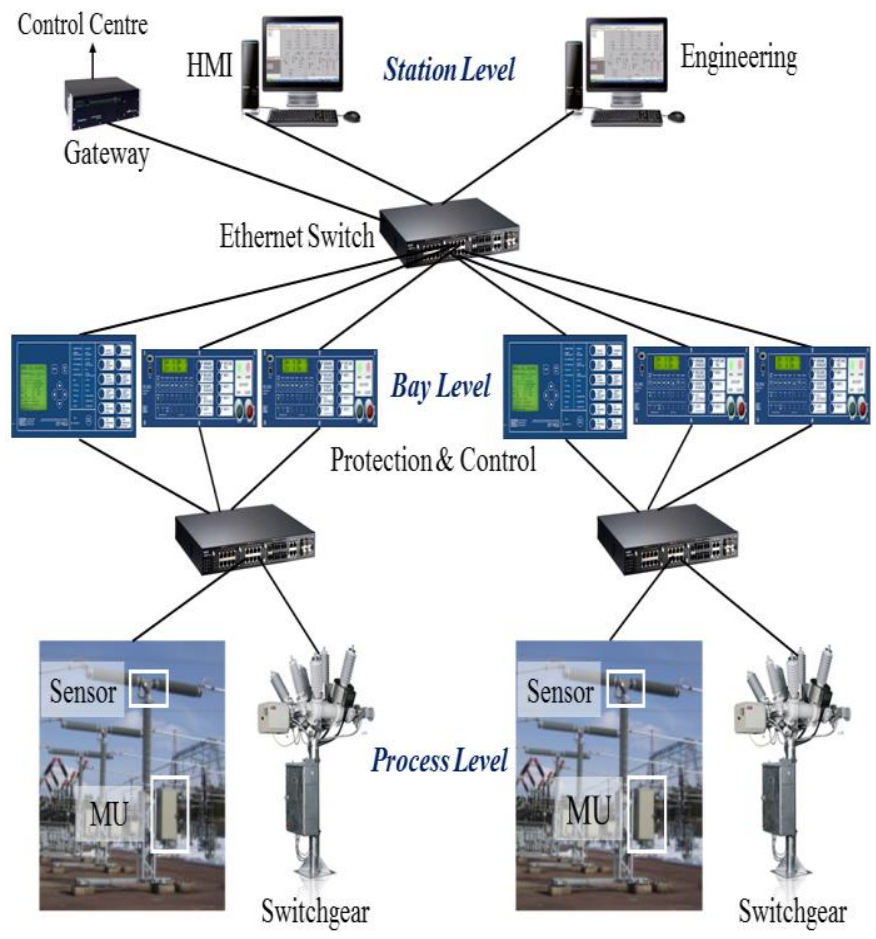

Fig. 1. Substation Automation System (SAS) architecture

provides an overview across the whole station and assure the supervision of all the substation equipment. This level includes Human Machine Interface (HMI) and engineering workstations as well as gateways to connect substation control centre to Wide Area Network (WAN). Bay level, includes protection and control IEDs of different bays such as circuit breakers, transformers, and capacitor banks. Equipment in bay level and station level are called secondary equipment. Process level, includes switchyard equipment, called primary equipment, such as $\mathrm{CTs} / \mathrm{PTs}$, remote I/O, actuators, merging units etc. The main purpose of this level is to acquire data from the electric processes and to make switching operations [13].

The communication of these three levels are carried out through the Process Bus and Station Bus. Process bus is the communication network which connects the IEDs at primary equipment level to other IEDs, such as MUs providing sampled measured values of current and voltage via the Local Area Network (LAN). Station Bus is the Communication network which inter-connects IEDs at Bay Level, IEDs at Station Level, and connects Bay Level to Station Level [13].

\section{IEC 61850 STANDARD}

The communication network is now considered the backbone of substation automation. Inappropriate configuration of the communication network may cause failure of substation automation functions and could make the applications at best inefficient and at worse ineffective. The 
IEC 61850 standard has enabled IEDs and devices in a substation to be integrated on a high-speed peer-to-peer communication network as well as client/server communication [2], [14].

In the IEC 61850 standard, protection and control functions are broken down into smaller units called Logical Nodes (LNs). In fact, these virtual units are the objects specified in the OO approach of the standard. Different functions in substations can be represented with these LNs. This is one of the most important advantages of the standard over legacy protocols [4], [15].

The dominant architectural construct that IEC 61850 adopts is the abstracting technique. This feature provides the definition of objects that are independent of any underlying communication protocols. In other words, "Abstract" means that the standard only determines what the services are intended to provide, rather than how they are built. Therefore, the system will be compatible with the future developments in the communication technology. Afterwards, the abstract model is mapped to a specific protocol stack that can meet the data and services requirements. Fig. 2 shows the five types of communication services provided by IEC 61850. Among

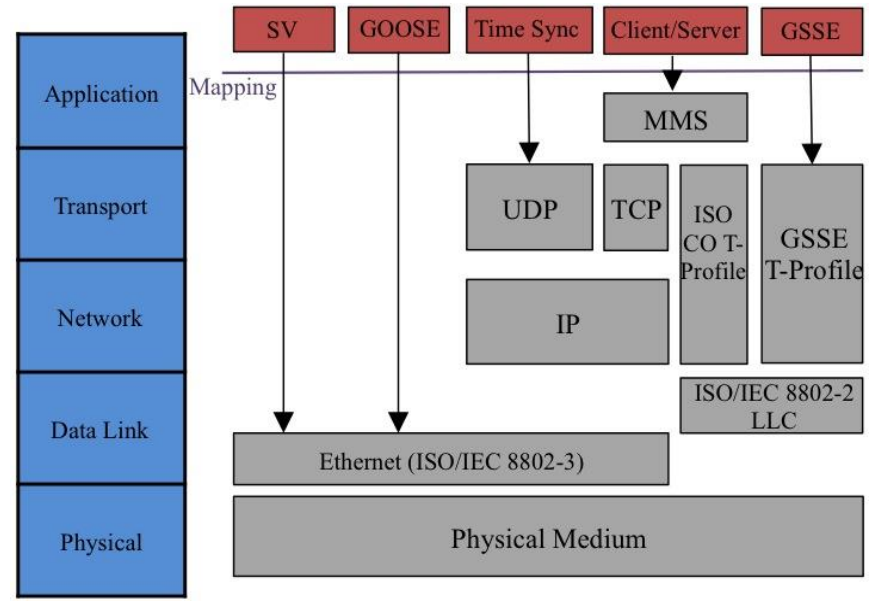

Fig. 2. Communication services defined in IEC 61850 them, Sampled Value (SV) and Generic Object Oriented Substation Event (GOOSE) are mapped directly to the Ethernet Link Layer. Therefore, they eliminate processing of any middle layers and increase the performance [16].

\section{SUbSTATION SimUlation}

The open source OMNeT++ discrete event simulator has been used to simulate the proposed substation architecture [5]. The INET framework, an open-source communication network simulation package for the OMNeT++, contains the common node models and protocols [17]. However, some required models were designed and configured that will be discussed.

The substation model that has been considered for simulation consists of one Transformer Bay and two Feeder Bays. It also includes Station PC and Server and all these nodes are connected via Ethernet Switch through star architecture. Fig. 3 shows the communications architecture of the simulated substation in OMNeT++ [5]. The Transformer Bay model has two Protection and Control IEDs, two Breakers, one MU, and also equipped with one PMU. The two Feeders have similar architecture, which consists of two Protection and Control IEDs, one Breaker, and one MU. These IEDs are also connected via Ethernet Switch through star architecture. The MU IEDs need to send Sampled Value messages so they modelled based on a three-layer communication protocol stack, which Application Layer packets are directly mapped to the Ethernet Link Layer. The Protection and Control IEDs may require to communicate with Station PC and Server using TCP/IP protocol, apart from receiving Sampled Values from the MUs and sending GOOSE Trip messages to the Breakers. Therefore, they should support both 5-layer Internet Protocol and 3-layer IEC 61850 communication protocol stacks. Therefore, a new node model has been designed for this purpose in OMNeT++, as shown in Fig. 4. This node model has two types of applications, TCP/IP and IEC 61850-based applications, which have a shared Link Layer. In the Link Layer a new
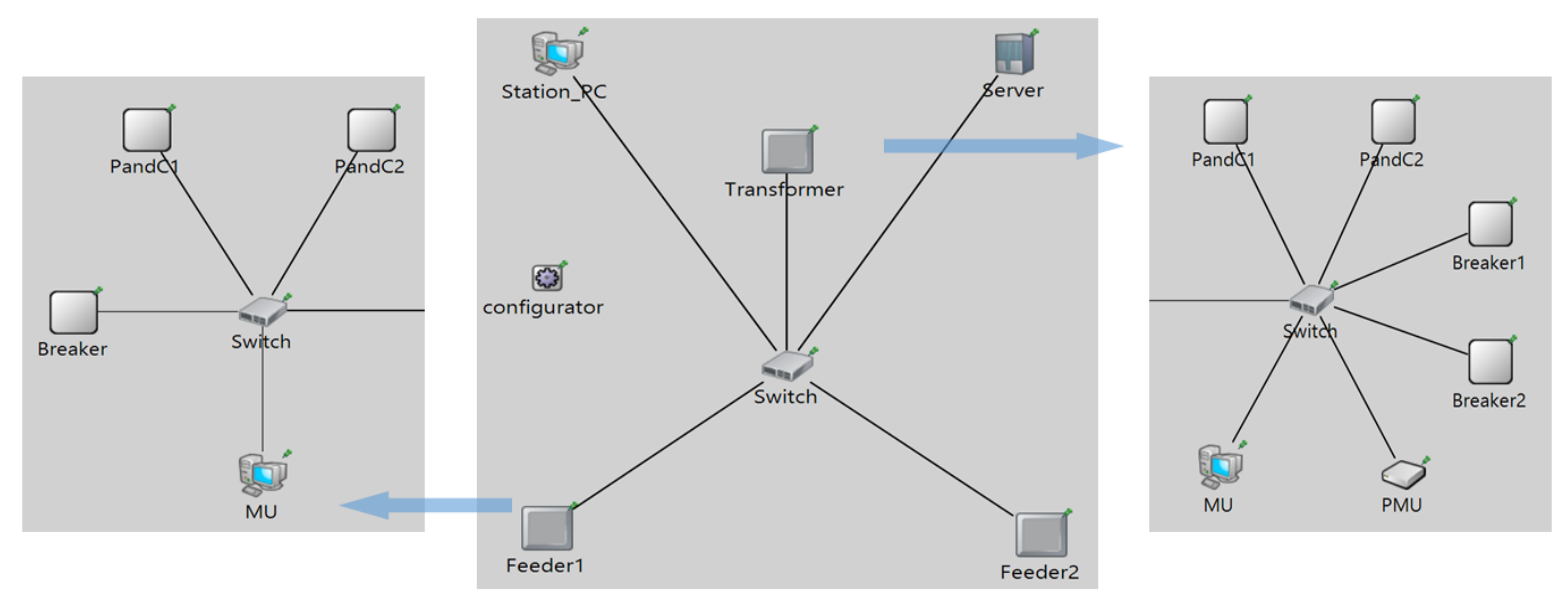

Fig. 3. The communications architecture of the simulated substation 


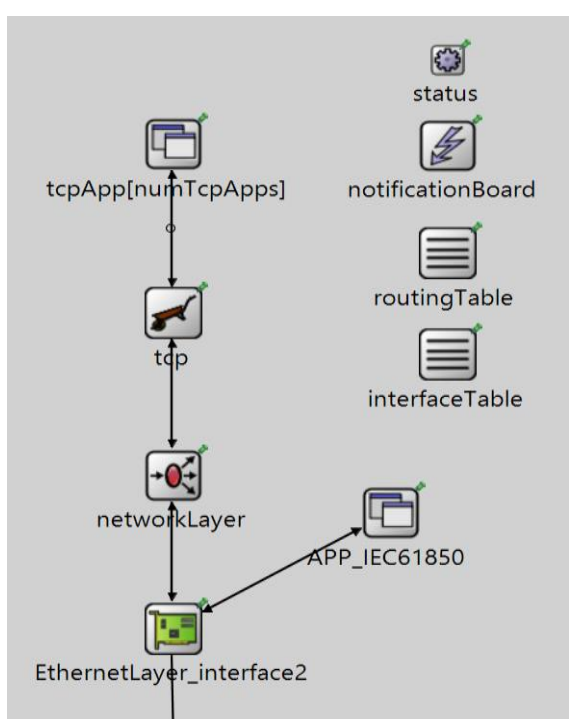

Fig. 4. IED model for TCP/IP and IEC 61850 communication

module has been designed, called Controller, to perform two required tasks. The first task is to provide two separate connections for the two applications packets arriving to the Queue and Ethernet Encapsulation modules. The second task is to classify the packet received by the node to determine whether it is a TCP/IP or IEC61850 packet so that the packet can be passed to the relevant Application Layer. A similar node model has been created for the Breaker IEDs, since they need to send status information to the Station Server based on TCP/IP as well as communicate with Protection and control IEDs to receive or send IEC 61850-based commands or status information.

The generated traffic by the nodes has been configured based on the following assumptions in accordance with Reference [18], [19]. The MUs send Sampled Value messages to the corresponding Protection and Control IEDs at sampling rate of $4800 \mathrm{~Hz}$. For the MUs inside the two Feeders this message size is 52 bytes, while for the Transformer Bay MU is 98 bytes. This is because the Transformer Bay MU contains two datasets of current and voltage.

The PMU IED generates 50 samples per second with the destination of Station Server. The PMU communicate based on User Datagram Protocol (UDP) with the message size of 50 bytes. Communication over network using Internet Protocol (IP) can be connection-oriented (TCP) or connectionless (UDP). TCP rearranges data packets in the order specified and retransmits lost or corrupted data. In the case of UDP there is no built-in ordering and recovery of data, but the transmission speed is higher than TCP. Therefore, currently UDP is used commonly with PMUs since a small amount of lost data is preferable over delayed data in real-time measurement [20]. The packet size depends on the number of synchrophasor parameters that a PMU measures. The 50 bytes message size has been chosen through capturing a real PMU packets by Wireshark, an open source packet analyser, and analysing the packets specifications [21].

Furthermore, all the Protection and Control IEDs and Breaker IEDs send update meter values or Breakers status information to the Station Server. This messages are sent at a rate of $20 \mathrm{~Hz}$ and size of 32 bytes using TCP/IP protocol. It has been assumed that a fault happens in Feeder1 and causes Protection and Control IEDs in Feeder 1 and Transformer Bay to send GOOSE Trip messages to corresponding Breakers. The Trip message size has been set to 16 bytes and is sent four times to ensure its delivery. One of the Protection and Control IEDs in Transformer Bay configured to send Trip messages continuously in order to introduce possible higher background traffic.

In the first scenario, 10Mbps LAN has been considered for all the substation communications and Table II and Table III show the obtained simulation EtE delay results for Sampled Value and Trip messages respectively. In the second scenario, the previous substation LAN has been replaced by $100 \mathrm{Mbps}$ LAN while the other network configurations and design are as previous scenario. The obtained results show a great improvement from the latency point of view as illustrated in Table IV and Table V.

TABLE II

SAMPLED VALUE DELAY

\begin{tabular}{|c|c|c|c|c|}
\hline \multirow{2}{*}{ Bay } & \multicolumn{4}{|c|}{$\begin{array}{c}\text { End-to-End Delay (ms) } \\
(10 \text { Mbps LAN) }\end{array}$} \\
\cline { 2 - 5 } & Average & Min & Max & STDEV \\
\hline Transformer & 0.2019 & 0.1985 & 0.2656 & 0.0119 \\
\hline Feeder 1 & 0.1488 & 0.1441 & 0.2108 & 0.0131 \\
\hline Feeder 2 & 0.1287 & 0.1249 & 0.1912 & 0.0138 \\
\hline
\end{tabular}

TABLE III

TRIP MESSAGE DELAY

\begin{tabular}{|c|c|c|c|c|}
\hline \multirow{2}{*}{ Bay } & \multicolumn{4}{|c|}{$\begin{array}{c}\text { End-to-End Delay (ms) } \\
\text { (10 Mbps LAN) }\end{array}$} \\
\cline { 2 - 5 } & Average & Min & Max & STDEV \\
\hline Transformer & 0.1225 & 0.1153 & 0.187 & 0.0167 \\
\hline Feeder 1 & 0.1275 & 0.1153 & 0.1734 & 0.0214 \\
\hline Interbay & 0.2618 & 0.2306 & 0.3937 & 0.0401 \\
\hline
\end{tabular}

TABLE IV

SAMPLED VALUE DELAY

\begin{tabular}{|c|c|c|c|c|}
\hline \multirow{2}{*}{ Bay } & \multicolumn{4}{|c|}{$\begin{array}{c}\text { End-to-End Delay (ms) } \\
\text { (100 Mbps LAN) }\end{array}$} \\
\cline { 2 - 5 } & Average & Min & Max & STDEV \\
\hline Transformer & 0.0206 & 0.0199 & 0.0266 & 0.0019 \\
\hline Feeder 1 & 0.0152 & 0.0145 & 0.0212 & 0.0018 \\
\hline Feeder 2 & 0.0130 & 0.0125 & 0.0189 & 0.0014 \\
\hline
\end{tabular}


TABLE V

TRIP MESSAGE DELAY

\begin{tabular}{|c|c|c|c|c|}
\hline \multirow{2}{*}{ Bay } & \multicolumn{4}{|c|}{$\begin{array}{c}\text { End-to-End Delay (ms) } \\
(100 \text { Mbps LAN) }\end{array}$} \\
\cline { 2 - 5 } & Average & Min & Max & STDEV \\
\hline Transformer & 0.0129 & 0.0116 & 0.0199 & 0.0025 \\
\hline Feeder 1 & 0.0122 & 0.0116 & 0.0173 & 0.0014 \\
\hline Interbay & 0.0273 & 0.0232 & 0.0399 & 0.0054 \\
\hline
\end{tabular}

Apart from the Sampled Value and Trip messages, delay of PMUs packets have also been analysed. In our analysis, we performed four scenarios to investigate the delay characteristics of the PMU inside the substation. The first scenario is the one as described already using UDP/IP protocol. For the second scenario we modelled PMU based on the created IEC 61850 node to communicate over Ethernet. In the second scenario the EtE delay of PMU packets reduced by $10 \%$ compared to the UDP/IP scenario. However, as a large portion of this EtE delay is due to the relatively heavy background traffic on the Station Server, a new server has been added to the Station Level of the substation to work as PDC. Then the third and fourth scenarios performed for more accurate comparison of the UDP/IP and IEC 61850-based PMU communication. From the obtained results as shown in Table VI, the Ethernet-based IEC 61850 communication reduced the PMU's packets delay by $27 \%$ compared to the UDP/IP protocol.

In SAS, the propagation delays in the physical links are much smaller compared to the other latency factors associated with protocols, acknowledgement messages, processing delays etc. This is merely due to the relatively short distances that the packets need to travel between communicating nodes in the network [8].

\section{CONCLUSIONS AND FURTHER WORK}

SAS is widely used in order to improve the reliability of power system. The success of a SAS relies heavily on the use of an effective communication system to link the various monitoring, control, and protection elements within a

TABLE VI

PMU DELAY

\begin{tabular}{|c|c|c|c|c|}
\hline \multirow{2}{*}{ Scenario } & \multicolumn{4}{|c|}{$\begin{array}{c}\text { End-to-End Delay (ms) } \\
\text { (100 Mbps LAN) }\end{array}$} \\
\cline { 2 - 5 } & Average & Min & Max & STDEV \\
\hline $\begin{array}{c}\text { Station Server } \\
\text { UDP/IP }\end{array}$ & 0.0602 & 0.0548 & 0.0666 & 0.0029 \\
\hline $\begin{array}{c}\text { Station Server } \\
\text { IEC 61850 }\end{array}$ & 0.0540 & 0.0455 & 0.0618 & 0.0037 \\
\hline $\begin{array}{c}\text { PDC Server } \\
\text { UDP/IP }\end{array}$ & 0.0265 & 0.0251 & 0.0317 & 0.0022 \\
\hline $\begin{array}{c}\text { PDC Server } \\
\text { IEC 61850 }\end{array}$ & 0.0192 & 0.0183 & 0.0249 & 0.0017 \\
\hline
\end{tabular}

substation. From the analysis carried out in this research, it can be observed that IEC 61850 based on Ethernet shown acceptable performance for substation communications. Furthermore, a preliminary studies performed to introduce and evaluate IEC 61850 protocol for PMUs applications. For the future work this simulated model of the substation communications network should be refined and improved from number of aspects. One of them is to use more accurate Application Layer and message format for IEC 61850-based communications, Sampled Value and GOOSE. Also VLAN should be configured in the Ethernet Layer in order to reduce the broadcast domain and limit it into the bay. In addition, the considered substation in this research has star architecture. A star architecture has a backbone switch that link all other switches. The advantage of star configuration is easy maintenance, flexible for expansion, and low delay. However, a failure of this switch will result in the entire communication system down. Other substation communications architecture that provide higher redundancy will be investigated as a further work.

\section{REFERENCES}

[1] M. S. Thomas, I. Ali, "Reliable, Fast, and Deterministic Substation Communication Network Architecture and its Performance Simulation," IEEE Transactions on Power Delivery, vol.25, no.4, pp.2364-2370, Oct. 2010.

[2] C. R. Ozansoy, A. Zayegh, A. Kalam, "The Application-View Model of the International Standard IEC 61850," Transactions on Power Delivery, vol.24, no.3, pp.1132-1139, July 2009.

[3] International Standard "IEC 61850: Communication networks and system for power utility automation - Part 2: Glossary," IEC, Dec. 2003.

[4] M. Golshani, G. A. Taylor, I. Pisica, P. Ashton, "Investigation of open standards to enable interoperable wide area monitoring for transmission systems," 47th International Universities Power Engineering Conference (UPEC), 4-7 Sept. 2012.

[5] OMNeT++ Network Simulation Framework, [www.omnetpp.org]

[6] M. Golshani, G. A. Taylor, I. Pisica, P. Ashton, "Laboratory- Based Deployment and Investigation of PMU and OpenPDC Capabilities" The 10th International Conference on AC and DC Power Transmission (IET) ACDC 2012, 4-6 December 2012.

[7] P. Myrda, K. Donahoe, "The true vision of automation," IEEE Power and Energy Magazine, vol.5, no.3, pp.32-44, May-June 2007.

[8] B. Falahati, M. J. Mousavi, M. Vakilian, "Latency considerations in IEC 61850-enabled Substation Automation Systems," IEEE Power and Energy Society General Meeting, 24-29 July 2011.

[9] K. Kaneda, S. Tamura, N. Fujiyama, Y. Arata, H. Ito, "IEC61850 based Substation Automation System," Joint International Conference on Power System Technology, POWERCON and IEEE Power India Conference, 12-15 Oct. 2008.

[10] S. Bricker, T. Gonen, L. Rubin, "Substation automation technologies and advantages," IEEE Computer Applications in Power, vol.14, no.3, pp.31-37, July 2001

[11] L. Zhu, D. Shi, X. Duan, "Standard Function Blocks for Flexible IED in IEC 61850-Based Substation Automation," IEEE Transactions on Power Delivery, vol.26, no.2, pp.1101-1110, April 2011.

[12] International Standard "IEC 61850: Communication networks and system for power utility automation - Part 7-1: Basic communication structure - Principles and models," IEC, Nov. 2011.

[13] International Standard "IEC 61850: Communication networks and system for power utility automation - Part 1: Introduction and overview," IEC, March. 2013.

[14] L. Zhu, D. Shi, P. Wang, "IEC 61850-Based Information Model and Configuration Description of Communication Network in Substation Automation," IEEE Transactions on Power Delivery, vol.29, no.1, pp.97-107, Feb. 2014. 
[15] S. Mohagheghi, J. C. Tournier, J. Stoupis, L. Guise, T. Coste, C. A. Andersen, J. Dall, "Applications of IEC 61850 in distribution automation," IEEE/PES Power Systems Conference and Exposition (PSCE), pp.1-9, 20-23 March 2011.

[16] R. E. Mackiewicz, "Overview of IEC 61850 and Benefits," IEEE PES Power Systems Conference and Exposition, pp.623-630, Oct. 29 Nov. 1 2006.

[17] INET Framework, [http://inet.omnetpp.org]

[18] T. S. Sidhu, Y. Yin, "Modelling and Simulation for Performance Evaluation of IEC61850-Based Substation Communication Systems," IEEE Transactions on Power Delivery, vol.22, no.3, pp.1482-1489, July 2007.

[19] J. Juarez, C. R. Morcillo, J. A. R. Mondejar, "Simulation of IEC 61850based substations under OMNeT++," In Proc. of the 5th Int. ICST Conference on Simulation Tools and Techniques, pp. 319-326, New York, Mar. 2012.

[20] M. Golshani, G. A. Taylor, I. Pisica, P. Ashton, "Implementation of Wide Area Monitoring Systems and Laboratory-Based Deployment of PMUs," 48th International Universities Power Engineering Conference (UPEC), 2-5 Sept. 2013.

[21] Wireshark, [http://www.wireshark.org] 